\title{
CONCEPCIONES ERRÓNEAS SOBRE ALIMENTACIÓN EN FUTUROS PROFESORES. CONSTRUCCIÓN DE CONOCIMIENTO PEDAGÓGICO
}

\author{
Future teachers' misconceptions about food. Building \\ pedagogical knowledge
}
Des conceptions erronées sur l'alimentation chez les futurs professeurs. Une construction de la connaissance pédagogique

Maximiliano Rodrigo Vega y José Manuel Ejeda Manzanera

Universidad Complutense. Facultad de Educación. c/ Rector Royo Villanova, s/n. 28040 Madrid. Correo-e: mrodrig1@edu.ucm.es, jmejeda@botmail.com

Fecha de recepción: enero de 2008

Fecha de aceptación definitiva: abril de 2008

BIBLID [(1130-3743) 20, 2008, 225-247]

RESUMEN

El presente estudio responde al interés de unir la investigación en la práctica educativa con el desarrollo del conocimiento pedagógico. En concreto, a partir de una investigación sobre la enseñanza de los conocimientos en alimentación en futuros profesores, se plantea una secuencia de niveles de concepciones que pueden constituir una guía de selección de contenidos educativos para trabajar en la Educación de la Alimentación.

Palabras clave: práctica educativa, conocimiento pedagógico, Educación para la Salud, formación del profesorado, Educación en Alimentación. 
MAXIMILIANO RODRIGO VEGA Y JOSÉ MANUEL EJEDA MANZANERA CONCEPCIONES ERRÓNEAS SOBRE ALIMENTACIÓN EN FUTUROS PROFESORES. CONSTRUCCIÓN DE CONOCIMIENTO PEDAGÓGICO

\section{SUMMARY}

The present study responds to the interest of uniting research in educational practice with the development of pedagogical knowledge. Specifically, based on research into the teaching of nutritional knowledge to future teachers, a sequence of concept levels is proposed which may serve as a guide for selecting educational content to work with in the area of Nutrition Education.

Key words: educational practice, pedagogical knowledge, Education for Health, teacher training, Nutrition Education.

\section{SOMMAIRE}

Cette étude répond à l'intérêt d'unir la recherche dans le domaine de la pratique éducative au développement de la connaissance pédagogique. À partir d'une recherche sur l'enseignement des connaissances en alimentation à des futurs professeurs, on y développe une séquence de niveaux de conception qui pourraient constituer un guide pour la sélection des contenus éducatifs à travailler dans l'Éducation à l'Alimentation.

Mots clef: pratique éducative, connaissance pédagogique, Éducation pour la Santé, formation des professeurs, Éducation et Alimentation.

\section{Justificación: la alimentación como temática Clave en Educación para la Salud}

En nuestros días promover acciones que potencien una alimentación saludable entre la ciudadanía se ha convertido en una cuestión de alto interés social. Baste recordar, en el ámbito español, la propuesta del programa NAOS (Nutrición, Actividad física y prevención de la Obesidad), promovido desde enero de 2005 por el Ministerio de Sanidad y Consumo junto con el Ministerio de Educación y Ciencia en colaboración con las Comunidades Autónomas, que recoge, entre otras, las siguientes acciones para el ámbito educativo:

Incluir en los cursos de Formación del Profesorado materiales didácticos y orientaciones sobre la alimentación y su incidencia sobre la salud...

Incluir en la formación de los alumnos conocimientos y habilidades relativos a la alimentación y la nutrición...

Vemos así reforzada nuestra idea de considerar la alimentación como temática clave de Educación para la Salud en la formación inicial o permanente del profesorado de enseñanza obligatoria, temática en la que venimos trabajando hace ya varios años y donde se enmarca el trabajo que ahora presentamos y que pretende 
dar un paso más allá, pues buscamos responder a esa demanda social con propuestas pedagógicas concretas procedentes de la investigación en la práctica educativa. Ésta es la razón que nos ha animado a publicar este trabajo en una revista de educación y, más concretamente, de teoría de la educación.

Y, expuesto así el interés prioritario que nos mueve, procede en primer lugar definir claramente el término "alimentación" y enfrentarlo al de nutrición. Apoyándonos en autores como Fernández-Crehuet y Pinedo (1988, 250), podemos decir que

La alimentación es la forma y manera de proporcionar al cuerpo humano las sustancias que le son indispensables para mantener la salud y la vida, por tanto es objeto claro de educación. Por el contrario, la nutrición se definiría como el conjunto de procesos fisiológicos por los cuales el cuerpo humano recibe, transforma y utiliza las sustancias contenidas en los alimentos que constituyen los materiales necesarios para mantener la vida.

Cubero (1998, 33), a su vez, entiende que

...con mucha frecuencia los términos alimentación y nutrición son utilizados como si fueran sinónimos, cuando en realidad describen dos procesos que, si bien se encuentran íntimamente ligados, son diferentes en muchos aspectos.

Según lo descrito, y desde un punto de vista pedagógico, la alimentación es claramente educable, pues podemos enseñar a elegir un tipo de alimento u otro; no así la nutrición, ya que nuestra fisiología es la que es y nuestra voluntad no la puede cambiar, pues una vez que el alimento ha entrado en nuestro cuerpo sigue su curso fisiológico.

Sin embargo, la alimentación como temática de Educación para la Salud (EpS), a pesar de su importancia social creciente, está poco integrada en la educación formal; de hecho, suelen estudiarse sobre todo cuestiones fisiológicas y, desde el punto de vista de la investigación educativa, las propuestas docentes ensayadas, concretamente en el entorno educativo español, están generalmente relacionadas con los niveles de Educación Primaria o Secundaria (Banet y Núñez, 1996; Membiella y Cid, 1998; Pérez de Eulate, 1992; Yus Ramos, 1995). En el ámbito de la formación del profesorado son todavía más escasas (Del Carmen, 1997; Membiella y Cid, 1998; Rodrigo, 1999 y 2000), a pesar del interés altamente estratégico que tendría formar al profesor en aspectos básicos de educación alimentaria. Si a ello añadimos que en EpS se considera (Young, 1995, 14) que "difícilmente se puede enseñar lo que apenas se conoce o no se practica", tendremos una justificación más de este trabajo.

Finalmente, y aunque la labor de educar para mejorar la alimentación implicaría trabajar diversos campos como el conceptual, el psicológico o el cultural (Gavidia, 1998), nosotros, como educadores de maestros, nos referiremos aquí esencialmente a la dimensión conceptual por considerarla nuclear, no exclusivo pero sí esencial, para sentar las bases de una mejor cultura alimentaria entre la población que va a recibir una educación básica. De manera que, si logramos hacer 
MAXIMILIANO RODRIGO VEGA Y JOSÉ MANUEL EJEDA MANZANERA CONCEPCIONES ERRÓNEAS SOBRE ALIMENTACIÓN EN FUTUROS PROFESORES. CONSTRUCCIÓN DE CONOCIMIENTO PEDAGÓGICO

propuestas fundamentadas pedagógicamente sobre cómo estructurar los conocimientos en alimentación para intentar mejorar la formación de los futuros profesores, estaremos llenando una laguna importante que en estos momentos muestra la literatura pedagógica.

\subsection{Presentación de la asignatura "La alimentación bumana en la escuela"}

Describiremos ahora de una manera general ${ }^{1}$ los objetivos y la estructura del programa de enseñanza implementado para la asignatura La alimentación bumana en la escuela ya que, como se apreciará más adelante, constituye uno de los fundamentos de este trabajo.

El diseño de esta asignatura siguió un proceso iniciado al realizar un estudio sobre preferencias en temas de Educación para la Salud (EpS) y sobre posibles maneras de abordarlas en los nuevos planes de estudio de Magisterio. Entre las conclusiones de dicho trabajo, se observó que la gran mayoría de los alumnos centraban su interés en la temática de la alimentación y solicitaban su integración en su formación (Rodrigo, 1995). Con el afán de satisfacer las necesidades formativas de los alumnos y dado que el concepto de "alimentación" está íntimamente relacionado con el "concepto nuclear de salud", en el curso 1997-98 se introdujo una nueva asignatura -La alimentación bumana en la escuela (Rodrigo, 1999 y 2000)-, que posteriormente se ha ido perfeccionando.

La materia, de 4,5 créditos, se imparte fundamentalmente en Magisterio, a estudiantes de 20 a 35 años de edad, de la Facultad de Educación de la Universidad Complutense de Madrid. Consideramos que son alumnos a los que se les ha dado ya nociones de los procesos involucrados en la nutrición (generalmente saben fisiología de la nutrición pero poca alimentación) en los cursos anteriores de Bachillerato o Educación Secundaria, donde han estudiado asignaturas de Biología o de Ciencias de la Naturaleza. En el Cuadro 1 se presenta un esquema general de su desarrollo, cuyos objetivos generales son los siguientes:

a) Aprendizaje de los principales conceptos relacionados con la temática.

b) La revisión crítica de las propias pautas de alimentación, y así permitir cambios en sus actitudes y hábitos alimentarios.

c) Ofrecer experiencias y modelos educativos que guarden relación con el tema y que puedan servir de referencia para su futura labor docente.

1. Puede consultarse con más detalle en Rodrigo (1999 y 2000). 
MAXIMILIANO RODRIGO VEGA Y JOSÉ MANUEL EJEDA MANZANERA

CONCEPCIONES ERRÓNEAS SOBRE ALIMENTACIÓN EN FUTUROS PROFESORES.

CONSTRUCCIÓN DE CONOCIMIENTO PEDAGÓGICO

CuAdro 1. Módulo teóriCo-PRÁCTICO de la asignatura:

LA ALIMENTACIÓN HUMANA EN LA ESCUELA (RODRIGO, 2000, ACTUALIZADO)

\begin{tabular}{|c|c|}
\hline Módulo Teórico (temas clave) & $\begin{array}{l}\text { Módulo Práctico (actividades guía: I, } \\
\text { individual; Pg, en grupos; G, de clase) }\end{array}$ \\
\hline $\begin{array}{l}\text { - I. Fundamentos conceptuales. } \\
\text { - I.0. Introducción. Significado biológico } \\
\text { de la Alimentación. }\end{array}$ & $\begin{array}{l}\text { - Intr.: Sesión de vídeo sobre la Alimenta- } \\
\text { ción y la Nutrición }(G) \text {. }\end{array}$ \\
\hline $\begin{array}{l}\text { - I.1. Principales nutrientes. (Hidratos de } \\
\text { Carbono, Lípidos, Proteínas, Sustancias } \\
\text { Minerales, Vitaminas y Agua). }\end{array}$ & $\begin{array}{l}\text { - I.1. ¿Qué nutrientes fundamentales tiene } \\
\text { un alimento como la leche?: Act. (Pg): } \\
\text { experimental en laboratorio. }\end{array}$ \\
\hline $\begin{array}{l}\text { - I.2. Principales necesidades nutritivas } \\
\text { humanas. (Energéticas. Plásticas-Cons- } \\
\text { tructoras. Reguladoras). }\end{array}$ & $\begin{array}{l}\text { - I.2. ¿Cuál es nuestra situación?: Act. } \\
\text { (I/G): cálculo de Pesos Ideales. ¿Cómo } \\
\text { conseguir lo necesario?: Act. (I): cálculo } \\
\text { de Biotipos Energéticos. }\end{array}$ \\
\hline $\begin{array}{l}\text { - I.3. La dieta equilibrada. (Grupos de ali- } \\
\text { mentos. Raciones. Dieta equilibrada). }\end{array}$ & $\begin{array}{l}\text { - I.3. ¿Qué comes?: Act. (I/G): registro } \\
\text { semanal de dietas. ¿Cómo mejorar la } \\
\text { situación?: Act. (I/G): Propuesta de } \\
\text { mejoras. }\end{array}$ \\
\hline $\begin{array}{l}\text { - II Cuestiones sociales y culturales. } \\
\text { (Sociología, Etología y Ecología de la } \\
\text { Alimentación. Preparación y conserva- } \\
\text { ción de alimentos. Toxiinfecciones). }\end{array}$ & $\begin{array}{l}\text { - II.1. ¿Qué cuestiones sociales pueden } \\
\text { interesarnos?: Act. (Pg): Lectura y } \\
\text { comentario de textos }\end{array}$ \\
\hline $\begin{array}{l}\text { - III Cuestiones educativas. Organización } \\
\text { de la enseñanza de la Alimentación. }\end{array}$ & $\begin{array}{l}\text { - III.1. ¿Cómo trabajar en el aula?: Act. } \\
\text { (Pg): Análisis de unidades didácticas. }\end{array}$ \\
\hline
\end{tabular}

\section{Planteamiento de la inVESTigación}

\subsection{Problemática y objetivos}

La investigación que aquí presentamos pretende definir los conocimientos que poseen los alumnos (futuros profesores) sobre los alimentos como base para mejorar su formación alimentaria y, en último término, desarrollar un conocimiento pedagógico sobre la enseñanza de la alimentación. Para muchos autores (Gavidia, 1998; Del Carmen y Martín, 1998; Rodrigo, 1999 y 2000), si el nivel de representación de los conceptos de alimentación y los alimentos es bajo, esto significaría que en su práctica profesional es posible que los profesores no atiendan aspectos importantes derivados de estos conocimientos, como creación de entornos favorecedores de conductas saludables y desarrollo de la capacidad de tomar decisiones informadas sobre cómo alimentarse adecuadamente. 
MAXIMILIANO RODRIGO VEGA Y JOSÉ MANUEL EJEDA MANZANERA CONCEPCIONES ERRÓNEAS SOBRE ALIMENTACIÓN EN FUTUROS PROFESORES. CONSTRUCCIÓN DE CONOCIMIENTO PEDAGÓGICO

\subsection{Diseño experimental}

El diseño fue cuasiexperimental, modalidad empleada dentro del ámbito de la investigación social y fácilmente interpretable (Ato, 1995; Campbell y Stanley, 1982; Gómez y Hombrados, 1988). El ejemplo prototipo queda representado en el cuadro siguiente, en el que una intervención a partir de la asignatura La alimentación en la escuela (X) busca generar cambio en los conocimientos de los alumnos sobre alimentación (Y). Asimismo, previo análisis de resultados, el grupo experimental se compara con el grupo control, por lo que se controla una posible intervención de otros factores que pudieran haber contribuido a la alteración de las puntuaciones u observaciones finales.

Cuadro 2. Esquema del diseño Seguido

\begin{tabular}{|l|c|c|c|}
\hline & \multicolumn{3}{|c|}{ Secuencia de registro } \\
\hline Grupos & Pretest & Tratamiento & Postest \\
Experimental (GE) & YE1 & $\mathrm{X}$ & YE2 \\
Control (GC) & YC1 & - & YC2 \\
\hline
\end{tabular}

\subsection{Instrumento de recogida de información y muestras de estudio}

Para avanzar en lo expuesto, se utilizó un cuestionario elaborado a partir de los propuestos por Rodrigo (1999, 2000) y por Banet y Núñez (1991), que se modificó con el fin de adaptarlo a la problemática planteada en el estudio. Se elaboró un instrumento previo, que se pasó a un grupo reducido de estudiantes, y cuyas respuestas permitieron ajustar la redacción de las preguntas a nuestros objetivos para cada uno de los contenidos que se pretenden estudiar. Se empleó un cuestionario de preguntas abiertas y cerradas, utilizando dos criterios de selección: que fueran fáciles de comprender y que contemplaran cuestiones consideradas clave para la formación personal de los estudiantes sobre alimentación. La versión definitiva de la parte del estudio al que nos referimos en este artículo se encuentra en el Anexo A.

El trabajo de campo se realizó con grupos (ver Tabla 1) de alumnos de la Facultad de Educación de la Universidad Complutense de Madrid durante los años 2001 al 2004. El grupo experimental (GE) en el Cuestionario Inicial y Final contaba con 147 alumnos y el grupo control (GC) se componía de 75 alumnos, que presentaron características de ambos géneros y edad comprendida entre 18 y 36 años. 
MAXIMILIANO RODRIGO VEGA Y JOSÉ MANUEL EJEDA MANZANERA

CONCEPCIONES ERRÓNEAS SOBRE ALIMENTACIÓN EN FUTUROS PROFESORES. CONSTRUCCIÓN DE CONOCIMIENTO PEDAGÓGICO

Tabla 1. Muestras de número de alumnos del estudio

\begin{tabular}{|l|c|c|c|c|}
\hline \multirow{2}{*}{$\begin{array}{l}\text { rrupos } \\
\text { Investigación }\end{array}$} & \multicolumn{2}{|c|}{$\begin{array}{c}\text { Cuestionario } \\
\text { Inicial (Pre) }\end{array}$} & \multicolumn{2}{c|}{$\begin{array}{c}\text { Cuestionario } \\
\text { Final (Post) }\end{array}$} \\
\hline Años & Experimental & Control & Experimental & Control \\
\hline $2001-02$ & 25 & 25 & 25 & 25 \\
$2002-03$ & 66 & 25 & 66 & 25 \\
$2003-04$ & 56 & 25 & 56 & 25 \\
Total n ${ }^{\circ}$ de & & & & 75 \\
alumnos & 147 & 75 & 147 & \\
\hline
\end{tabular}

En concreto, en el grupo control (GC) la edad de los sujetos estaba comprendida entre los 18 y los 34 años, con una media de 20,88 años, una mediana de 20 y una moda de 19. En el caso del grupo experimental (GE) la edad se sitúa entre los 18 años y los 36 años. La media es de 20,96 años, con una moda de 19 y una mediana de 20. En conjunto predominan las mujeres sobre los varones (95,24\% de mujeres frente a $4,76 \%$ de hombres).

Para garantizar la homogeneidad de las variables básicas, se realizó una prueba de análisis estadístico U de Mann Whitney (prueba no paramétrica) sobre los datos del Cuestionario Inicial (CI), entre el Grupo Experimental (GE) y el Grupo Control (GC), utilizándose el programa estadístico SPSS 11.6 para Windows, obteniéndose una significación de la prueba (U de Mann Whitney > 0,05), comprobándose así la similitud entre ambos grupos.

\subsection{Análisis de resultados}

La forma de puntuación que se empleó en el cuestionario es la que nosotros mismos establecimos de acuerdo con el cuerpo de conocimientos teóricos sobre alimentación (véase el comienzo de cada apartado de resultados). Previo al análisis de los resultados del Grupo Experimental, se realizó un control estadístico de una posible intervención de factores externos (como ya señalamos) que pudieran o no haber contribuido a la alteración de las puntuaciones finales en cada una de las cuestiones planteadas. Para ello se realizó la Prueba de Wilcoxon (muestras pareadas) con el Grupo Control antes y después de la acción didáctica para cada una de las cuestiones, utilizando el programa estadístico SPSS 11.6 para Windows, lo que nos permitió determinar la causalidad del tratamiento en la variable observada, al no presentar cambios significativos dicho Grupo Control. Posteriormente, se analizaron los resultados globales de cada pregunta utilizando la Prueba de Wilcoxon (muestras pareadas) para determinar si existieron "cambios significativos" en los conocimientos de los alumnos con respecto a las diferentes cuestiones, 
MAXIMILIANO RODRIGO VEGA Y JOSÉ MANUEL EJEDA MANZANERA CONCEPCIONES ERRÓNEAS SOBRE ALIMENTACIÓN EN FUTUROS PROFESORES. CONSTRUCCIÓN DE CONOCIMIENTO PEDAGÓGICO

utilizando el programa estadístico SPSS 11.6 para Windows, y valorando dicha prueba de la siguiente forma:

a. Si la significación asintótica (Sig. asintót.) bilateral $>0,05$ entonces se acepta Ho (no ha habido variación por efecto de la acción didáctica).

b. Si la significación asintótica (Sig. asintót.) bilateral $\leq 0,05$ entonces se acepta $\mathrm{Ha}$ (hipótesis alternativa, es decir, existió variación por efecto de la docencia).

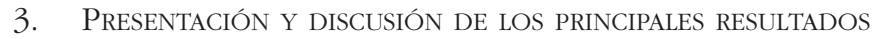

3.1. Cuestión primera (¿Qué son y qué diferencias existen entre alimentación y nutrición?)

Cuadro 3. Valoración de las diferentes contestaciones a la Cuestión Primera

\begin{tabular}{|c|c|c|}
\hline Rango & Contestación & Valor \\
\hline \multirow{10}{*}{$0-8$} & No responden o responden de forma incoherente & 0 \\
\hline & Definen $\mathrm{Al}=\mathrm{Nu}$ sin incluir diferencias & 0 \\
\hline & Definen de forma sencilla $\mathrm{Al} \neq \mathrm{Nu}$ sin incluir diferencias & 1 \\
\hline & Definen de forma compleja $\mathrm{Al} \neq \mathrm{Nu}$ sin incluir diferencias & 2 \\
\hline & Diferencian Al-Nu con los términos voluntario y consciente & 3 \\
\hline & Diferencian Al-Nu con el término educable & 4 \\
\hline & Definen de forma sencilla $\mathrm{Al} \neq \mathrm{Nu}$ y Diferencian $\mathrm{Al}-\mathrm{Nu}$ con términos** & 5 \\
\hline & Definen de forma compleja $\mathrm{Al} \neq \mathrm{Nu}$ y Diferencian $\mathrm{Al}-\mathrm{Nu}$ con términos ${ }^{* * *}$ & 6 \\
\hline & Definen de forma sencilla $\mathrm{Al} \neq \mathrm{Nu}$ y Diferencian $\mathrm{Al}-\mathrm{Nu}$ con el término* & 7 \\
\hline & Definen de forma compleja $\mathrm{Al} \neq \mathrm{Nu}$ y Diferencian $\mathrm{Al}-\mathrm{Nu}$ con el término* & 8 \\
\hline
\end{tabular}

*educable; ${ }^{* *}$ consciente y voluntario; $\mathrm{Al}$ = Alimentación; $\mathrm{Nu}=$ Nutrición.

Se puede observar a partir de la Tabla 2 que en el Cuestionario Inicial (CI) el $80,27 \%$ de los alumnos confunden los conceptos de alimentación y nutrición, aspecto que está en línea con la observación planteada por autores como Cubero (1988, 33), mientras que en el Cuestionario Final (CF) se reduce al 21,09\%. Consideramos de interés que los alumnos, como futuros educadores, manejen el concepto de alimentación con precisión, dado que, como ya indicamos, la alimentación (como acto voluntario) es objeto claro de educación. En cuanto a si los cambios producidos en los alumnos sobre dicha cuestión son significativos, podemos observar en el Cuadro 4 que se acepta la hipótesis alternativa ( $\mathrm{Ha}$ ) de que hay diferencias significativas después de la acción didáctica; no obstante, persisten diversidad de concepciones dentro del alumnado. Esto demuestra que las 
MAXIMILIANO RODRIGO VEGA Y JOSÉ MANUEL EJEDA MANZANERA CONCEPCIONES ERRÓNEAS SOBRE ALIMENTACIÓN EN FUTUROS PROFESORES.

CONSTRUCCIÓN DE CONOCIMIENTO PEDAGÓGICO

concepciones de los alumnos (futuros profesores) sobre la alimentación se manifestarían inicialmente como muy inmaduras para abordar cualquier programa de enseñanza de esta temática, aunque con el efecto de asignaturas como la que aquí nos referimos evolucionan bastante positivamente, si bien lo hacen con matices que vamos a ver seguidamente.

Tabla 2. Datos de valoraciones dadas aplicando lo dicho en el Cuadro 3 y SEgún las CONTESTACiONES del Cuestionario Inicial (CI) y Final (CF) A LA CUESTIÓN PRIMERA

\begin{tabular}{|c|c|c|c|c|}
\hline Contestación & \multicolumn{2}{|c|}{ Número de Alumnos } & \multicolumn{2}{c|}{ Porcentaje de Alumnos } \\
\hline Valor & $\begin{array}{c}\text { Cuestionario } \\
\text { Inicial }\end{array}$ & $\begin{array}{c}\text { Cuestionario } \\
\text { Final }\end{array}$ & $\begin{array}{c}\text { Cuestionario } \\
\text { Inicial }\end{array}$ & $\begin{array}{c}\text { Cuestionario } \\
\text { Final }\end{array}$ \\
\hline 0 & 118 & 31 & $80,27 \%$ & $21,09 \%$ \\
\hline 1 & 10 & 14 & $6,80 \%$ & $9,52 \%$ \\
\hline 2 & 14 & 19 & $9,52 \%$ & $12,93 \%$ \\
\hline 3 & 2 & 18 & $1,36 \%$ & $12,24 \%$ \\
\hline 4 & 3 & 23 & $2,04 \%$ & $15,65 \%$ \\
\hline 5 & 0 & 14 & $0,00 \%$ & $9,52 \%$ \\
\hline 6 & 0 & 10 & $0,00 \%$ & $6,80 \%$ \\
\hline 7 & 0 & 11 & $0,00 \%$ & $7,48 \%$ \\
\hline 8 & 0 & 7 & $0,00 \%$ & $4,76 \%$ \\
\hline Total & 147 & 147 & $100 \%$ & $100 \%$ \\
\hline
\end{tabular}

Cuadro 4. Estadística de Contraste entre el Cuestionario inicial (Ci) y FinAl (CF) DE LA TABLA 2

Estadísticos de contraste (b)

\begin{tabular}{|l|c|c|c|}
\hline $\begin{array}{l}\text { Cuestionario } \\
\text { Inicial-Final (CI-CF) }\end{array}$ & $\mathrm{Z}$ & $\begin{array}{c}\text { Sig. asintót. } \\
\text { (bilateral) }\end{array}$ & $\begin{array}{c}\text { Aceptación } \\
\text { Hipótesis }\end{array}$ \\
\hline $\begin{array}{l}\text { Diferencia entre } \\
\text { Alimentación y } \\
\text { Nutrición }\end{array}$ & $\begin{array}{c}8,947(\mathrm{a}) \\
-\end{array}$ &, 000 & Ha \\
\hline
\end{tabular}

a Basado en los rangos negativos $(\mathrm{CF}>\mathrm{CI})$.

b Prueba de los rangos con signo de Wilcoxon. 


\subsection{Cuestión segunda}

a. Valoración global a la pregunta: ¿Qué nutrientes fundamentales presentan los alimentos energéticos, plásticos y reguladores?

Cuadro 5. VAloración de las difEREnTEs Contestaciones a CAda Apartado DE LA CUESTIÓN SEGUNDA (VÉASE ANEXO A)

\begin{tabular}{|c|c|c|c|c|c|}
\hline Cuestión & Apartado & Concepto & Rango & Contestación & Valor \\
\hline \multirow{18}{*}{ Segunda } & \multirow{6}{*}{ Primero } & \multirow{6}{*}{$\begin{array}{l}\text { Conocimientos sobre } \\
\text { qué nutrientes } \\
\text { contienen los } \\
\text { alimentos energéticos }\end{array}$} & \multirow{6}{*}{$0-3$} & Carbohidratos y lípidos & 3 \\
\hline & & & & Carbohidratos o lípidos & 2 \\
\hline & & & & Carbohidratos y otros & 1 \\
\hline & & & & Lípidos y otros & 1 \\
\hline & & & & Resto de respuestas & 0 \\
\hline & & & & No contesta & 0 \\
\hline & \multirow{6}{*}{ Segundo } & \multirow{6}{*}{$\begin{array}{l}\text { Conocimientos } \\
\text { sobre qué nutrientes } \\
\text { contienen los } \\
\text { alimentos plásticos }\end{array}$} & \multirow{6}{*}{$0-3$} & Proteínas y minerales & 3 \\
\hline & & & & Proteínas o minerales & 2 \\
\hline & & & & Proteínas y otros & 1 \\
\hline & & & & Minerales y otros & 1 \\
\hline & & & & Resto de respuestas & 0 \\
\hline & & & & No contesta & 0 \\
\hline & \multirow{6}{*}{ Tercero } & \multirow{6}{*}{$\begin{array}{l}\text { Conocimientos } \\
\text { sobre qué nutrientes } \\
\text { contienen los } \\
\text { alimentos reguladores }\end{array}$} & \multirow{6}{*}{$0-3$} & Vitaminas y minerales & 3 \\
\hline & & & & Vitaminas o minerales & 2 \\
\hline & & & & Vitaminas y otros & 1 \\
\hline & & & & Minerales y otros & 1 \\
\hline & & & & Resto de respuestas & 0 \\
\hline & & & & No contesta & 0 \\
\hline
\end{tabular}


MAXIMILIANO RODRIGO VEGA Y JOSÉ MANUEL EJEDA MANZANERA CONCEPCIONES ERRÓNEAS SOBRE ALIMENTACIÓN EN FUTUROS PROFESORES. CONSTRUCCIÓN DE CONOCIMIENTO PEDAGÓGICO

Tabla 3. Datos de valoraciones globales dadas aplicando lo Dicho en el Cuadro 4 y Según las contestaciones al Cuestionario Inicial (CI) Y FiNAL (CF) DE LA CUESTIÓN SEGUNDA

\begin{tabular}{|c|c|c|c|c|}
\hline Contestación & \multicolumn{2}{|c|}{ Número de Alumnos } & \multicolumn{2}{c|}{ Porcentaje de Alumnos } \\
\hline Valor & $\begin{array}{c}\text { Cuestionario } \\
\text { Inicial }\end{array}$ & $\begin{array}{c}\text { Cuestionario } \\
\text { Final }\end{array}$ & $\begin{array}{c}\text { Cuestionario } \\
\text { Inicial }\end{array}$ & $\begin{array}{c}\text { Cuestionario } \\
\text { Final }\end{array}$ \\
\hline 0 & 12 & 2 & $8,16 \%$ & $1,36 \%$ \\
\hline 1 & 16 & 3 & $10,88 \%$ & $2,04 \%$ \\
\hline 2 & 21 & 11 & $14,29 \%$ & $7,48 \%$ \\
\hline 3 & 29 & 11 & $19,73 \%$ & $7,48 \%$ \\
\hline 4 & 24 & 10 & $16,33 \%$ & $6,80 \%$ \\
\hline 5 & 13 & 5 & $8,84 \%$ & $3,40 \%$ \\
\hline 6 & 7 & 21 & $4,76 \%$ & $14,29 \%$ \\
\hline 7 & 13 & 26 & $8,84 \%$ & $17,69 \%$ \\
\hline 8 & 5 & 26 & $3,40 \%$ & $17,69 \%$ \\
\hline 9 & 7 & 32 & $4,76 \%$ & $21,77 \%$ \\
\hline Total & 147 & 147 & $100 \%$ & $100 \%$ \\
\hline
\end{tabular}

Cuadro 6. Estadística de Contraste entre el Cuestionario Inicial (Ci) y Final (CF) DE LA TABLA 3

Estadísticos de contraste (b)

\begin{tabular}{|lc|c|c|}
\hline $\begin{array}{l}\text { CI-CF } \\
\text { Alimentos }\end{array}$ & Z & $\begin{array}{c}\text { Sig. asintót. } \\
\text { (bilateral) }\end{array}$ & $\begin{array}{c}\text { Aceptación } \\
\text { Hipótesis }\end{array}$ \\
\hline $\begin{array}{l}\text { Energéticos (E) } \\
\begin{array}{l}\text { Plásticos (P) } \\
\text { Reguladores (R) }\end{array}\end{array}$ & $-8,138(\mathrm{a})$ &, 000 & Ha \\
\hline
\end{tabular}

a Basado en los rangos negativos $(\mathrm{CF}>\mathrm{CI})$.

b Prueba de los rangos con signo de Wilcoxon.

De la Tabla 3 y del Cuadro 6 se desprende una mejora estadísticamente significativa en las concepciones de los estudiantes al asociar nutrientes con alimentos dietéticamente energéticos, plásticos o reguladores, pero persisten diversidad e concepciones poco maduras, como analizaremos con más detalle seguidamente. 
MAXIMILIANO RODRIGO VEGA Y JOSÉ MANUEL EJEDA MANZANERA CONCEPCIONES ERRÓNEAS SOBRE ALIMENTACIÓN EN FUTUROS PROFESORES. CONSTRUCCIÓN DE CONOCIMIENTO PEDAGÓGICO

b. Resultados parciales correspondientes a la cuestión segunda

Tabla 4. Relación totall de N. ${ }^{\circ}$ De CONTESTACiones dadas (CON Respecto al Menos a UN NUTRIENTE) A LA PREGUNTA "LOS ALIMENTOS ENERGÉTICOS CONSTAN SOBRE TODO DE..." (LAS MARCADAS * SERÍAN LAS ACERTADAS, EL RESTO SON ERRÓNEAS)

\begin{tabular}{|l|c|c|}
\hline \multicolumn{3}{|c|}{ Número total de contestaciones recibidas (los energéticos tienen...) } \\
\hline Nutriente mayoritario & Cuestionario Inicial & Cuestionario Final \\
\hline Carbohidratos* & $102^{*}$ & $125^{*}$ \\
\hline Lípidos* & $44^{*}$ & $92^{*}$ \\
\hline Proteínas & 72 & 31 \\
\hline Vitaminas & 40 & 10 \\
\hline Minerales & 11 & 4 \\
\hline
\end{tabular}

TAbla 5. Relación total del n. ${ }^{\circ}$ De CONTESTACiOnes dadas (CON RESPECTO Al MENOS A UN NUTRIENTE) A LA PREGUNTA: "LOS ALIMENTOS PLÁSTICOS CONSTAN SOBRE TODO DE..." (LAS MARCADAS * SERÍAN LAS ACERTADAS)

\begin{tabular}{|l|c|c|}
\hline \multicolumn{3}{|c|}{ Número total de contestaciones recibidas (los plásticos tienen...) } \\
\hline Nutriente mayoritario & Cuestionario Inicial & Cuestionario Final \\
\hline Proteínas* & $61^{*}$ & $121^{*}$ \\
\hline Minerales* & $55^{*}$ & $79^{*}$ \\
\hline Carbohidratos & 32 & 15 \\
\hline Lípidos & 63 & 31 \\
\hline Vitaminas & 27 & 25 \\
\hline
\end{tabular}

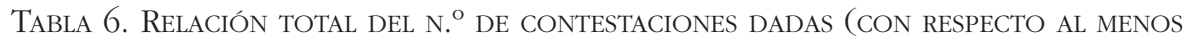
A UN NUTRIENTE) A LA PREGUNTA: "LOS ALIMENTOS REGULADORES CONSTAN SOBRE TODO DE..." (LAS MARCADAS* SERÍAN LAS ACERTADAS)

\begin{tabular}{|l|c|c|}
\hline \multicolumn{3}{|c|}{ Número total de contestaciones recibidas (los reguladores tienen...) } \\
\hline Nutriente mayoritario & Cuestionario Inicial & Cuestionario Final \\
\hline Vitaminas* & $77^{*}$ & $107^{*}$ \\
\hline Minerales* & $72^{*}$ & $99^{*}$ \\
\hline Carbohidratos & 14 & 6 \\
\hline Lípidos & 19 & 4 \\
\hline Proteínas & 18 & 6 \\
\hline
\end{tabular}


MAXIMILIANO RODRIGO VEGA Y JOSÉ MANUEL EJEDA MANZANERA

De la revisión de los resultados parciales procedentes de las contestaciones a la cuestión segunda (Tablas 4, 5 y 6) se obtienen consideraciones como las siguientes:

- Dentro de los "alimentos energéticos" (Tabla 4), los nutrientes más conocidos son los carbohidratos. Sorprende cómo inicialmente asocian a las proteínas como fuente de energía, descartando casi por completo el papel de los lípidos, que juegan un papel incluso mayor que el de los carbohidratos por su aporte de $9 \mathrm{kcal}$ por gramo (frente a 4 de los carbohidratos). Similares observaciones se han recogido en estudios con alumnos de Educación Secundaria (Banet y Núñez, 1997a y b).

- Los "alimentos plásticos" (Tabla 5) son los peor asociados a nutrientes previo a la acción didáctica, como ya hemos puesto en evidencia en otros estudios (Rodrigo, 2000); además el término plástico puede estar en la base de la confusión de la inclusión de los lípidos como tales por la apariencia "plástica de las grasas o lípidos". Por ello, plantearíamos utilizar, en aras a mejoras didácticas en la educación alimentaria, términos dietéticos como "constructores o estructurales".

- Los "alimentos reguladores" (Tabla 6) suelen asociarse bastante bien con sus nutrientes mayoritarios, especialmente vitaminas, aspecto también manifestado por autores como Banet y Núñez (1997a y b).

Podemos resumir que, con la formación recibida, los alimentos reguladores y los energéticos son los que mejor asocian los alumnos a nutrientes claves en ellos presentes, no así los "alimentos plásticos o mejor dicho constructores". Sin embargo, aunque el aprendizaje mejora notablemente, persisten aún en algunos estudiantes las concepciones iniciales confusas especialmente en estos últimos. 
MAXIMILIANO RODRIGO VEGA Y JOSÉ MANUEL EJEDA MANZANERA CONCEPCIONES ERRÓNEAS SOBRE ALIMENTACIÓN EN FUTUROS PROFESORES. CONSTRUCCIÓN DE CONOCIMIENTO PEDAGÓGICO

\subsection{Cuestión tercera}

a. Valoración global a la pregunta: ¿Cuál es la función y el nutriente principal que presentan determinados alimentos?

CUAdRO 7. VALORACiÓN DE LAS DifERENTES CONTESTACIONES SOBRE LA "FUNCIÓN Y NUTRIENTE PRINCIPAL" DE DETERMINADOS ALIMENTOS

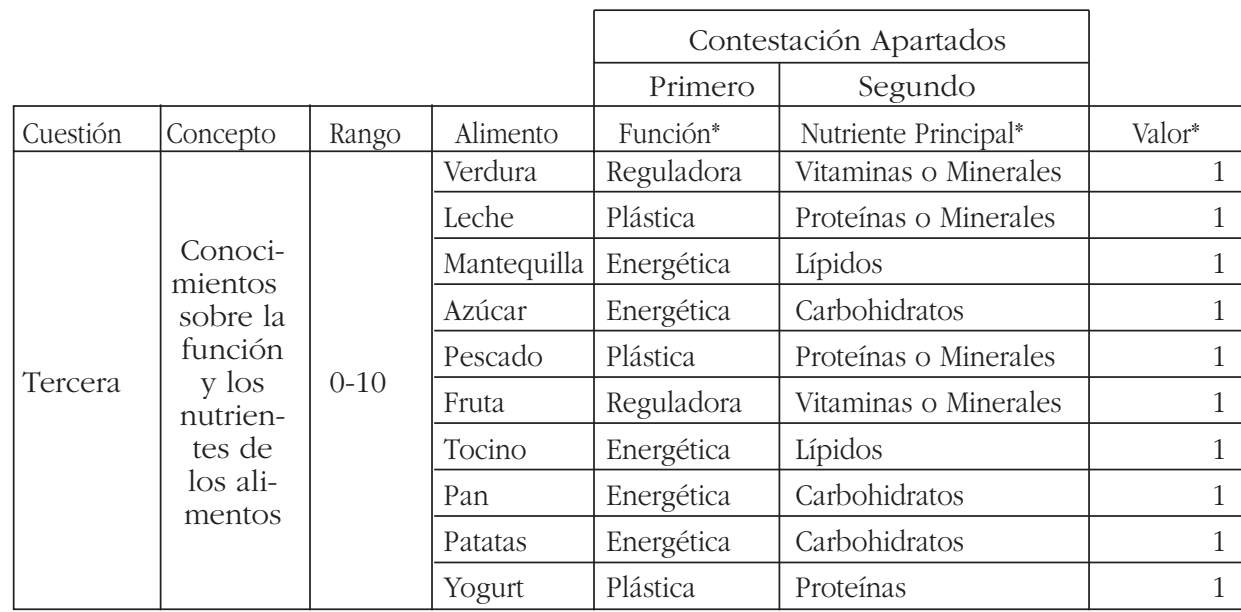

* Otras respuestas en cada uno de los apartados se valoran como 0.

Tabla 7. Datos de valoraciones globales dadas, aplicando lo dicho en el Cuadro 7 Y SEGÚN LAS CONTESTACIONES AL CUESTIONARIO INICIAL Y FINAL SOBRE LA «FUNCIÓN Y EL NUTRIENTE PRINCIPAL” DE DETERMINADOS ALIMENTOS

\begin{tabular}{|c|c|c|c|c|}
\hline Contestación & \multicolumn{2}{|c|}{ Número de Alumnos } & \multicolumn{2}{|c|}{ Porcentaje de Alumnos } \\
\hline Valor & C. Inicial & C. Final & C. Inicial $\%$ & C. Final $\%$ \\
\hline 0 & 12 & 5 & 8,16 & 3,40 \\
1 & 16 & 2 & 10,88 & 1,36 \\
2 & 20 & 9 & 13,61 & 6,12 \\
3 & 26 & 6 & 17,69 & 4,08 \\
4 & 20 & 13 & 13,61 & 8,84 \\
5 & 16 & 10 & 10,88 & 6,80 \\
6 & 10 & 17 & 6,80 & 11,56 \\
7 & 7 & 19 & 4,76 & 12,93 \\
8 & 7 & 26 & 4,76 & 17,69 \\
9 & 9 & 19 & 6,12 & 12,93 \\
10 & 4 & 21 & 2,72 & 14,29 \\
Total & 147 & 147 & 100 & 100 \\
\hline
\end{tabular}


MAXIMILIANO RODRIGO VEGA Y JOSÉ MANUEL EJEDA MANZANERA CONCEPCIONES ERRÓNEAS SOBRE ALIMENTACIÓN EN FUTUROS PROFESORES. CONSTRUCCIÓN DE CONOCIMIENTO PEDAGÓGICO

Cuadro 8. Estadística de Contraste entre el Cuestionario inicial (Ci) Y FiNAL (CF) DE LA TABLA 7

Estadísticos de contraste (b)

\begin{tabular}{|lc|c|c|}
\hline $\begin{array}{l}\text { CI-CF } \\
\text { Función-Nutriente }\end{array}$ & $Z$ & $\begin{array}{l}\text { Sig. asintót. } \\
\text { (bilateral) }\end{array}$ & $\begin{array}{c}\text { Aceptación } \\
\text { Hipótesis }\end{array}$ \\
\hline Alimentos & $-8,165(\mathrm{a})$ &, 000 & Ha \\
\hline
\end{tabular}

a Basado en los rangos negativos $(\mathrm{CF}>\mathrm{CI})$.

b Prueba de los rangos con signo de Wilcoxon.

Se puede observar, a partir de la Tabla 7, que en el Cuestionario Inicial (CI) un $74,83 \%$ de los alumnos consiguen cinco aciertos o menos (de diez posibles), es decir, existe bastante desconocimiento a la hora de precisar la asociación "tipo de alimento, su nutriente principal, su función dietética desempeñada", mientras que en el Cuestionario Final (CF) se reducen al 30,60\% los sujetos que puntúan menos de cinco. Consideramos clave que los alumnos manejen la asociación conceptual citada, dado que es la base de saber elegir conscientemente un tipo de alimento u otro (y, por tanto, también saber enseñar a elegirlos). En cuanto a si los cambios producidos en los alumnos sobre dicha cuestión son significativos, podemos observar en el Cuadro 8 que se acepta la hipótesis alternativa (Ha) de que hay diferencias significativas después de la acción didáctica; no obstante, pasamos a revisar algunos datos parciales, dado su interés para sacar conclusiones pedagógicas, como luego veremos.

b. Análisis de datos parciales de las contestaciones dadas por los alumnos a la cuestión tercera (ver Anexo A)

b1. Contestaciones que relacionan "alimento con nutriente principal en él presente"

TABla 8. Contestaciones (EN \%) RECiBidas a «QUÉ NUTRIENTE CONSIDERAN PRINCIPAL EN ALIMENTOS MAYORITARIAMENTE CONFORMADOS POR CARBOHIDRATOS* (CONTESTACIÓN CORRECTA)"

\begin{tabular}{|l|c|c|c|c|c|c|}
\cline { 2 - 7 } \multicolumn{1}{c|}{} & \multicolumn{9}{c|}{ Alimentos } \\
\hline & \multicolumn{2}{c|}{ Azúcar } & \multicolumn{2}{c|}{ Pan } & \multicolumn{2}{c|}{ Patatas } \\
\hline Nutriente principal & CI \% & CF $\%$ & CI \% & CF $\%$ & CI \% & CF \% \\
\hline Carbohidratos* & 60,54 & 72,79 & 71,43 & 82,99 & 63,95 & 73,47 \\
\hline Lípidos & 16,33 & 8,84 & 5,44 & 3,40 & 6,80 & 1,36 \\
\hline Proteínas & 6,80 & 6,80 & 10,20 & 3,40 & 15,65 & 11,56 \\
\hline Vitaminas & 2,04 & 0,68 & 1,36 & 1,36 & 2,04 & 6,80 \\
\hline Minerales & 10,88 & 4,76 & 8,16 & 4,76 & 8,84 & 3,40 \\
\hline
\end{tabular}


MAXIMILIANO RODRIGO VEGA Y JOSÉ MANUEL EJEDA MANZANERA CONCEPCIONES ERRÓNEAS SOBRE ALIMENTACIÓN EN FUTUROS PROFESORES. CONSTRUCCIÓN DE CONOCIMIENTO PEDAGÓGICO

Podemos observar en la Tabla 8 cómo con el azúcar, el pan y las patatas los alumnos no tienen ningún problema en englobarlos inicialmente como alimentos que contienen carbohidratos, con un porcentaje del 60\%, 71\% y del 63\% respectivamente, aunque después de la acción didáctica, en el Cuestionario Final, se obtienen valores incrementados en todos ellos.

TABla 9. CONTESTACIONES (EN \%) RECIBIDAS A "QUÉ NUTRIENTE CONSIDERAN PRINCIPAL EN ALIMENTOS MAYORITARIAMENTE CONFORMADOS POR LÍPIDOS* (CONTESTACIÓN CORRECTA)»

\begin{tabular}{|l|c|c|c|c|}
\cline { 2 - 5 } \multicolumn{1}{c|}{} & \multicolumn{3}{c|}{ Mlimentos } \\
\cline { 2 - 5 } \multicolumn{1}{c|}{} & \multicolumn{2}{c|}{ Mantequilla } & \multicolumn{2}{c|}{ Tocino } \\
\hline Nutriente principal & CI \% & CF $\%$ & CI \% & CF $\%$ \\
\hline Lípidos* & 61,90 & 78,23 & 74,15 & 82,99 \\
\hline Carbohidratos & 21,09 & 6,12 & 16,33 & 7,48 \\
\hline Proteínas & 10,20 & 8,16 & 5,44 & 6,12 \\
\hline Vitaminas & 0,68 & 1,36 & 0,00 & 0,00 \\
\hline Minerales & 2,72 & 1,36 & 1,36 & 0,68 \\
\hline
\end{tabular}

En la Tabla 9 se aprecia cómo con la mantequilla y el tocino los alumnos no tienen ningún problema en englobarlos inicialmente como alimentos que contienen lípidos, con un porcentaje del $62 \%$ y del $74 \%$ respectivamente, aunque después de la acción didáctica en el Cuestionario Final se obtienen valores incrementados en todos ellos.

TABLA 10. CONTESTACIONES (EN \%) RECIBIDAS A "QUÉ NUTRIENTE CONSIDERAN PRINCIPAL EN ALIMENTOS MAYORITARIAMENTE CONFORMAdOS POR ProteÍNAS* O Minerales* (AMBAS DADAS COMO CORRECTAS)"

\begin{tabular}{|l|c|c|c|c|c|c|}
\cline { 2 - 7 } \multicolumn{1}{c|}{} & \multicolumn{6}{c|}{ Alimentos } \\
\hline & \multicolumn{2}{c|}{ Leche } & \multicolumn{2}{c|}{ Pescado } & \multicolumn{2}{c|}{ Yogur } \\
\hline Nutriente principal & CI \% & CF $\%$ & CI \% & CF \% & CI \% & CF \% \\
\hline Proteínas* & 48,98 & 62,59 & 63,27 & 84,35 & 38,78 & 57,14 \\
\hline Minerales* & 11,56 & 7,48 & 14,29 & 6,12 & 8,84 & 8,84 \\
\hline Carbohidratos & 4,76 & 7,48 & 6,12 & 2,04 & 8,16 & 5,44 \\
\hline Lípidos & 9,52 & 3,40 & 2,72 & 1,36 & 10,88 & 3,40 \\
\hline Vitaminas & 19,73 & 11,56 & 8,84 & 4,08 & 27,21 & 19,73 \\
\hline
\end{tabular}

Se aprecia también en la Tabla 10 cómo con el pescado los alumnos no tienen ningún problema en englobarlo inicialmente como alimento que contiene 
especialmente proteínas. Respecto a la leche y el yogur existen algunos problemas de asignación de nutrientes mayoritarios. Después de la acción didáctica, en el Cuestionario Final, se obtienen valores incrementados en todos ellos, pero persiste la presencia de inclusión de vitaminas con cerca de un 20\% para el yogur y un $12 \%$ para la leche. Además, los alumnos tienen preferencia por las proteínas mejor que los minerales respecto de estos alimentos; recordemos la importancia dietética que se da al calcio como mineral clave aportado por los lácteos para prevenir la osteoporosis.

TABla 11. CONTESTACIONES (EN \%) RECIBIDAS A "QuÉ NUTRIENTE CONSIDERAN PRINCIPAL EN ALIMENTOS MAYORITARIAMENTE CONFORMADOS POR VITAMINAS* O MiNERALES*

(AMBAS DADAS COMO CORRECTAS)"

\begin{tabular}{|l|c|c|c|c|}
\cline { 2 - 5 } \multicolumn{1}{c|}{} & \multicolumn{3}{c|}{ Alimentos } \\
\cline { 2 - 5 } \multicolumn{1}{c|}{} & \multicolumn{2}{c|}{ Verdura } & \multicolumn{2}{c|}{ Fruta } \\
\hline Nutriente principal & CI \% & CF $\%$ & CI \% & CF \% \\
\hline Vitaminas* $^{*}$ & 53,74 & 62,59 & 81,63 & 91,84 \\
\hline Minerales* & 21,77 & 25,85 & 5,44 & 3,40 \\
\hline Carbohidratos & 3,40 & 2,04 & 2,72 & 2,04 \\
\hline Lípidos & 2,72 & 0,00 & 1,36 & 0,00 \\
\hline Proteínas & 14,97 & 6,80 & 4,76 & 0,68 \\
\hline
\end{tabular}

Se pone en evidencia en la Tabla 11 cómo tanto con la verdura como con la fruta los alumnos no tienen ningún problema en englobarlos inicialmente como alimentos que contienen vitaminas (mejor que minerales). Después de la acción didáctica, en el Cuestionario Final, se obtienen valores incrementados en cada uno de ellos.

Resumiendo, la asociación "alimento y nutriente principal en él presente" suele ser alta ya inicialmente y mejora con la docencia en todos los casos estudiados. Consideramos que el trabajo de la educación formal en este aspecto es bastante efectivo. Un caso especial sería la poca asociación de los minerales, especialmente del calcio, como nutriente clave en los lácteos a pesar de su importancia o la asociación errónea de las vitaminas como nutriente mayoritario que algunos alumnos asocian a alimentos como el yogur frente al contenido en calcio. A no ser que desconozcan que el calcio es un mineral y lo asignen como una vitamina (fruto de sobreexposición cultural a anuncios, por ejemplo), no tiene mucha explicación. 
MAXIMILIANO RODRIGO VEGA Y JOSÉ MANUEL EJEDA MANZANERA CONCEPCIONES ERRÓNEAS SOBRE ALIMENTACIÓN EN FUTUROS PROFESORES. CONSTRUCCIÓN DE CONOCIMIENTO PEDAGÓGICO

b2. Contestaciones que relacionan «alimento con función dietética desempeñada por él"

TABla 12A. CONTESTACIONES (EN \%) SOBRE FunCiÓN DIETÉTICA ASIGNADA A ALIMENTOS CUYA CUALIDAD ES CLARAMENTE ENERGÉTICA* (ACIERTO)

\begin{tabular}{|l|c|c|c|c|c|c|}
\cline { 2 - 7 } \multicolumn{1}{c|}{} & \multicolumn{6}{c|}{ Alimentos } \\
\hline Alimento & \multicolumn{2}{c|}{ Azúcar } & \multicolumn{2}{c|}{ Pan } & \multicolumn{2}{c|}{ Patatas } \\
\hline Función dietética & CI \% & CF \% & CI \% & CF \% & CI \% & CF \% \\
\hline Energéticos* & 79,59 & 86,39 & 50,34 & 74,83 & 51,02 & 63,95 \\
\hline Plásticos & 11,56 & 5,44 & 31,97 & 10,88 & 32,65 & 12,24 \\
\hline Reguladores & 6,80 & 4,76 & 12,93 & 11,56 & 13,61 & 19,05 \\
\hline
\end{tabular}

TABLA 12B. CONTESTACIONES (EN \%) SOBRE FUNCIÓN DIETÉTICA ASIGNADA A ALIMENTOS CUYA CUALIDAD ES CLARAMENTE ENERGÉTICA* (ACIERTO)

\begin{tabular}{|l|c|c|c|c|}
\cline { 2 - 5 } \multicolumn{1}{c|}{} & \multicolumn{3}{c|}{ Alimentos } \\
\cline { 2 - 5 } \multicolumn{1}{c|}{} & \multicolumn{2}{c|}{ Mantequilla } & \multicolumn{2}{c|}{ Tocino } \\
\hline Función dietética & CI \% & CF $\%$ & CI \% & CF \% \\
\hline Energéticos* & 48,98 & 59,86 & 49,66 & 73,47 \\
\hline Plásticos & 41,50 & 35,37 & 41,50 & 19,73 \\
\hline Reguladores & 6,80 & 2,72 & 6,12 & 2,04 \\
\hline
\end{tabular}

Respecto de la función dietética asignada a alimentos considerados claramente energéticos (azúcar, pan, patatas, mantequilla y tocino, ver Tablas12a y 12b), los alumnos inicialmente reconocen al azúcar sin ningún tipo de problemas, el pan y las patatas lo hacen con mayor dificultad, pero donde existen mayores confusiones es en la mantequilla y tocino. Esto puede asimilarse a lo que ocurría con la cuestión segunda (apartado 3.2) donde los lípidos o grasas eran menos conocidos como energéticos y se asociaban erróneamente como plásticos, como ya indicamos (además estos nutrientes están muy presentes en la mantequilla y tocino). Después de la acción didáctica, aunque el aprendizaje mejora significativamente, persisten aún en algunos estudiantes las concepciones iniciales erróneas, sobre todo con la mantequilla y el tocino, y su persistencia a seguir considerándolos alimentos con función plástica (Rodrigo, 1999 y 2000). Consideramos que la expresión "alimento plástico" encierra componentes de educación formal, pero también de educación no formal; en este sentido el término plástico asociado a alimentos moldeables como tocino y mantequilla, o de nutrientes viscosos como las grasas o lípidos, sería poco afortunado. 
MAXIMILIANO RODRIGO VEGA Y JOSÉ MANUEL EJEDA MANZANERA CONCEPCIONES ERRÓNEAS SOBRE ALIMENTACIÓN EN FUTUROS PROFESORES. CONSTRUCCIÓN DE CONOCIMIENTO PEDAGÓGICO

TABla 13. CONTESTACIONES (EN \%) SOBRE FUnCIÓN DIETÉTICA ASIGNADA A ALIMENTOS CUYA CUALIDAD ES CLARAMENTE PLÁSTICA* (ACIERTO)

\begin{tabular}{|l|c|c|c|c|c|c|}
\cline { 2 - 7 } \multicolumn{1}{c|}{} & \multicolumn{6}{c|}{ Alimentos } \\
\hline & \multicolumn{2}{|c|}{ Leche } & \multicolumn{2}{c|}{ Pescado } & \multicolumn{2}{c|}{ Yogur } \\
\hline Función dietética & CI \% & CF $\%$ & CI \% & CF $\%$ & CI \% & CF \% \\
\hline Plásticos* & 40,14 & 78,23 & 31,97 & 77,55 & 34,01 & 72,11 \\
\hline Energéticos & 44,22 & 14,29 & 22,45 & 11,56 & 21,77 & 8,16 \\
\hline Reguladores & 14,29 & 5,44 & 43,54 & 9,52 & 40,14 & 17,01 \\
\hline
\end{tabular}

En las respuestas sobre la función que los alumnos asignan a los alimentos considerados dietéticamente como plásticos (leche, pescado y yogur, ver Tabla 13), los alumnos inicialmente tienen dudas y los engloban indiscriminadamente en las tres funciones, aunque mayoritariamente tienden a encuadrar a dichos alimentos fuera de su función, englobándolos en energéticos (leche) y reguladores (pescado y yogur). Esto puede ser debido, al igual que en la cuestión segunda (apartado 3.2), a la denominación plástico (que crea confusión) y al desconocimiento de qué nutrientes mayoritarios contienen los alimentos plásticos (proteínas o minerales especialmente). Después de la acción didáctica, aunque el aprendizaje mejora significativamente, persisten aún en algunas concepciones iniciales erróneas (sobre todo con el yogur, al que siguen describiendo muchos como alimento regulador; sin duda la interferencia de la educación no formal con anuncios publicitarios donde se vende este producto como un "regulador del intestino" puede ser parte de la explicación). De nuevo, resaltamos el hecho de que consideramos que hoy día la alimentación está de moda y especialmente "en el desarrollo de los conceptos como alimento energético, plástico o regulador" participan componentes procedentes de la educación extraescolar (anuncios, reportajes...) que pueden interferir en lo que queremos transmitir los educadores.

TABla 14. Contestaciones (EN \%) SOBRE FunCiÓN DietÉTICA ASIGNADA A ALIMENTOS CUYA CUALIDAD ES CLARAMENTE REGULADORA* (ACIERTO)

\begin{tabular}{|l|c|c|c|c|}
\cline { 2 - 5 } \multicolumn{1}{c|}{} & \multicolumn{4}{c|}{ Alimentos } \\
\cline { 2 - 5 } \multicolumn{1}{c|}{} & \multicolumn{2}{c|}{ Verdura } & \multicolumn{2}{c|}{ Fruta } \\
\hline Función dietética & CI \% & CF \% & CI \% & CF \% \\
\hline Reguladores* & 85,03 & 87,07 & 76,19 & 88,44 \\
\hline Energéticos & 9,52 & 5,44 & 13,61 & 8,16 \\
\hline Plásticos & 4,76 & 6,80 & 8,16 & 3,40 \\
\hline
\end{tabular}


MAXIMILIANO RODRIGO VEGA Y JOSÉ MANUEL EJEDA MANZANERA CONCEPCIONES ERRÓNEAS SOBRE ALIMENTACIÓN EN FUTUROS PROFESORES. CONSTRUCCIÓN DE CONOCIMIENTO PEDAGÓGICO

Podemos observar en la Tabla 14 cómo con la verdura y la fruta los alumnos no tienen ningún problema en englobarlos inicialmente en reguladores con un porcentaje del $85 \%$ y el 76\% respectivamente, aunque después de la acción didáctica, en el Cuestionario Final, se obtienen valores incrementados sobre todo en la fruta (88\%), hasta alcanzar ambos casi el mismo porcentaje final.

Resumiendo, en la revisión de las contestaciones que asocian "alimento con función dietética desempeñada por él» los alumnos tienden a conocer mejor los alimentos reguladores, pero existe cierto grado de confusión entre las funciones energéticas y en especial las plásticas de los alimentos. En concreto, el término "plástico" ligado a alimentación creemos que es desafortunado y proponemos utilizar "constructor".

Como reflexión final, podemos considerar que los alumnos tienden a contestar mejor (cuestión tercera, véase lo descrito en 3.3) cuando se les pregunta qué nutrientes contienen determinados alimentos (verdura, leche, mantequilla...) que cuando se les pregunta (cuestión segunda, véase el apartado 3.2) de forma general qué nutrientes contienen los alimentos energéticos, plásticos o reguladores. Ésta y otras observaciones creemos que son relevantes en orden a propuestas pedagógicas sobre la alimentación que seguidamente resumimos.

\section{DisCUSIÓN PEDAGÓGICA: NIVELES EN FORMACIÓN ALIMENTARIA}

En base al análisis anterior pasamos a exponer las siguientes consideraciones:

Primera. Resaltamos que, inicialmente, la imprecisión en muchos conceptos implicaría que los alumnos estudiados (futuros profesores) no eran aptos para educar sobre alimentación, pero pasarían a obtener mejoras significativas conceptuales con asignaturas del tipo visto; por eso reclamamos para futuros profesores, que deben ejercer en la sociedad actual, algún tipo de formación alimentaria similar a la que hemos analizado.

Segunda. Asimismo, consideramos clave que los futuros profesores manejen la asociación conceptual «tipo de alimento, su nutriente principal en él presente, y función dietética desempeñada por su consumo", dado que está en la base de saber elegir conscientemente un tipo de alimento u otro (y, por tanto, también de saber educar sobre alimentación). Con respecto a esta asociación, se constata que los alumnos estudiados contestan mejor cuando se les pregunta qué nutrientes contienen determinados alimentos (verdura, leche, mantequilla...) que cuando se les pregunta de forma general qué nutrientes contienen los alimentos considerados dietéticamente energéticos, plásticos o reguladores, y, además, desconocen en determinados casos qué alimentos son energéticos, plásticos o reguladores, pero no así el nutriente principal que presentan.

Tercera. La observación anterior es relevante en orden a configurar una propuesta síntesis sobre la posible construcción del conocimiento pedagógico en alimentación; así, según la edad y cultura la información alimentaria relevante se podría configurar en los siguientes niveles: 
MAXIMILIANO RODRIGO VEGA Y JOSÉ MANUEL EJEDA MANZANERA

CONCEPCIONES ERRÓNEAS SOBRE ALIMENTACIÓN EN FUTUROS PROFESORES.

CONSTRUCCIÓN DE CONOCIMIENTO PEDAGÓGICO

- Primer nivel de formación. Se debería centrar en el conocimiento de los alimentos (que es lo cercano y palpable) y sus distintas agrupaciones según su frecuencia, diaria/semanal, y cantidades de consumo deseables.

- Segundo nivel de formación. Se trataría de avanzar en el conocimiento de los nutrientes mayoritarios de los alimentos (conceptos que implican procesos de enseñanza más formal).

- Tercer nivel de formación. Se profundizaría en el concepto de la función dietética (energética, constructora, reguladora) desempeñada por los alimentos en relación con los nutrientes mayoritarios que en cada alimento estén presentes (proceso de enseñanza más formal y plagado de interferencias culturales; así, como hemos indicado anteriormente, proponemos sustituir el término plástico por constructor).

- Cuarto nivel de formación. Se centraría en el estudio de las relaciones entre los nutrientes y las funciones de los mismos en el organismo (conceptos más formales de enseñanza de nutrición y fisiología).

Los alimentos tienen un significado mayor, principalmente social y familiar, para los alumnos, lo que no sucede con los nutrientes ni con su función dietética. Esta secuencia se fundamenta en las observaciones sobre conocimientos en alimentación que hemos ido viendo y se podría aplicar según vaya subiendo el nivel educativo en la enseñanza de la alimentación. En formación de profesores sería deseable llegar hasta el tercer nivel, dado que el cuarto sería más propio de profesionales de la salud. La mejora conceptual, que aunque a veces se consigue no es tan espectacular como desearíamos, estaría en la base para obtener mejoras alimentarias. Precisamente éste es otro aspecto en el que ahora seguimos trabajando.

Para concluir, queremos manifestar nuestro agradecimiento al alumnado que ha cursado la asignatura La alimentación humana en la escuela y ha participado en esta investigación, pues con su interés e ilusión nos ha seguido motivando para trabajar e investigar sobre algo tan actual y con tanto interés social como la mejora de la educación en alimentación.

\section{BibliografíA}

Ato, M. (1995) Tipología de los diseños cuasiexperimentales, en Anguera, M. T.; Arnau, J.; Ato, M.; Martínez, R.; Pascual, J. y Vallejo, G. (eds.). Métodos de investigación en Psicologia. Madrid, Síntesis.

BANET, E. y NúÑEZ, F. (1991) Estudio de los alimentos: Plan de actuación basado en una secuencia constructivista del aprendizaje, Investigación en la Escuela, 13, 31-58.

- (1996) Actividades en el aula para la reestructuración de ideas: un ejemplo relacionado con la nutrición humana, Investigación en la Escuela, 28, 52-59.

- (1997a) Students' conceptual patterns of human nutrition, International Journal of Science Education, 19 (5), 509-525.

- (1997b) Teaching and learning about human nutrition: a constructivist approach, International Journal of Science Education, 19 (10), 1169-1194. 
MAXIMILIANO RODRIGO VEGA Y JOSÉ MANUEL EJEDA MANZANERA CONCEPCIONES ERRÓNEAS SOBRE ALIMENTACIÓN EN FUTUROS PROFESORES. CONSTRUCCIÓN DE CONOCIMIENTO PEDAGÓGICO

Campbell, D. T. y Stanley, J. C. (1982) Diseños experimentales y cuasiexperimentales en la investigación social. Buenos Aires, Amorrortu.

Cubero, R. (1998) Aprendizaje de la digestión en la enseñanza primaria, Alambique, 16, 33-43.

Del Carmen, L. (1997) Educación para la Salud y hábitos alimentarios en la Formación Inicial del Profesorado de Educación Infantil y Primaria, en BANET, E. y Pro, A. (eds.). Actas $V$ Congreso Internacional sobre Investigación en Didáctica de las Ciencias. Murcia, Universidad de Murcia, 23-24.

Del Carmen, L. y Martín, J. M. (1998) Educación Para La Salud y Hábitos Alimentarios en la Formación Inicial del Profesorado de Educación Infantil y Primaria, en BANET, E. y PRO, A. (coords.). Investigación e Innovación en la Enseñanza de las Ciencias, vol. I. Lleida, Universidad de Valencia, 115-118.

EJEDA, J. M. (2006) El conocimiento sobre Alimentación en la Formación Inicial de Maestros. Trabajo de investigación de final de Programa de Doctorado (inédito). Madrid, Facultad de Educación-UCM.

Fernández-Creuhet, J. y Pinedo, A. (1988) Alimentación, Nutrición y Salud Pública, en PieDROLA, G. (coord.). Medicina Preventiva y Salud Pública. Barcelona, Salvat, pp. 250-260.

GAVIDIA, V. (1998) Una propuesta de formación del profesorado en educación para la salud como respuesta a sus necesidades profesionales, en BANET, E. y PRO, A. (coords.). Investigación e Innovación en la Enseñanza de las Ciencias, vol. II. Lleida, Universidad de Valencia, pp. 75-83.

Gómez, L. y Hombrados, I. (1988) Diseños de intervención comunitaria, en Martín, A.; Chacón, F. y Martínez, M. (eds.). Psicología Comunitaria. Madrid, Visor, 149-166.

Membiela, P. y Cid, M. C. (1998) Desarrollo de una unidad didáctica centrada en la alimentación humana, social y culturalmente contextualizada, Enseñanza de las Ciencias, 18 (3), 494-511.

Pérez de Eulate, L. (1992) Utilización de los conceptos previos de los alumnos en la enseñanza-aprendizaje de conocimientos de Biología. La nutrición humana: una propuesta de cambio conceptual. Tesis Doctoral. Bilbao, Universidad del País Vasco.

Rodrigo, M. (1995) Concepciones de los futuros profesores de primaria sobre Educación para la Salud, Revista Interuniversitaria de Formación del Profesorado, 24, 173-180.

- (1999) Puesta en práctica de una asignatura de alimentación en Formación Inicial del profesorado, Revista Complutense de Educación, 10 (1), 379-391.

- (2000) Una asignatura de alimentación en Formación Inicial del Profesorado, en Martín, M. (coord.). Reflexiones sobre la Didáctica de las Ciencias Experimentales. Actas de los XIX Encuentros de Didáctica de las Ciencias Experimentales. Madrid, Departamento de Didáctica de las Ciencias Experimentales-UCM, 268-274.

Young, I. (1995) La Educación para la salud en el ámbito educativo. Madrid, MEC-MSC. Yus Ramos, R. (1995) Ciencias de la Naturaleza II. Zaragoza, MEC-Edelvives. 
ANEXo A

Parte del cuestionario utilizada en la elaboración de este artículo (Cuestionario completo en Ejeda, 2006, 119-122).

\section{DATOS DE IDENTIFICACIÓN}

Apellidos y Nombre..

$\begin{array}{lllll}\text { Cuatrimestre: } & 1^{\circ} \mathrm{C} \quad 2^{\circ} \mathrm{C} \quad \text { Especialidad: } \\ & \text { Turno: } & 13: 30 \mathrm{~h} \quad 15: 00 \mathrm{~h}\end{array}$

CUESTIÓN 1: ¿Qué diferencias existen entre alimentación y nutrición?

CUESTIÓN 2: A continuación te presentamos una serie preguntas cuyas contestaciones corresponden a sustancias para la vida y que están presentes en los alimentos:

2.1. Los alimentos energéticos constan sobre todo de (señala DOS como máximo):

Proteínas

Minerales

Carbohidratos

Lípidos

Vitaminas

2.2. Los alimentos plásticos o estructurales constan sobre todo de (señala DOS como máximo):

$\square$ Proteínas $\square$ Minerales $\square$ Carbohidratos $\square$ Lípidos $\quad \square$ Vitaminas

2.3. Los alimentos reguladores constan sobre todo de (señala DOS como máximo):

$\square$ Proteínas $\square$ Minerales $\square$ Carbohidratos $\square$ Lípidos $\square$ Vitaminas

CUESTIÓN 3: Seguidamente te proponemos una lista de alimentos SEÑALA, según proceda, en las CASILLAS una característica muy peculiar de CADA ALIMENTO

\begin{tabular}{|c|c|c|}
\hline Alimentos & $\begin{array}{l}\text { 3.1. Indica si dietéticamente es } \\
\text { fundamentalmente: Energé- } \\
\text { tico/Plástico/Regulador }\end{array}$ & $\begin{array}{l}\text { 3.2. Señala una sustancia mayoritaria pre- } \\
\text { sente en el alimento: Carbohidratos/ } \\
\text { Lípidos/Proteínas/Vitaminas/Minerales }\end{array}$ \\
\hline \multicolumn{3}{|l|}{ Verduras } \\
\hline \multicolumn{3}{|l|}{\begin{tabular}{|l} 
Leche \\
\end{tabular}} \\
\hline \multicolumn{3}{|l|}{ Mantequilla } \\
\hline \multicolumn{3}{|l|}{ Azúcar } \\
\hline \multicolumn{3}{|l|}{ Pescado } \\
\hline \multicolumn{3}{|l|}{ Fruta } \\
\hline \multicolumn{3}{|l|}{ Tocino } \\
\hline \multicolumn{3}{|l|}{ Pan } \\
\hline \multicolumn{3}{|l|}{ Patatas } \\
\hline Yogur & & \\
\hline
\end{tabular}


